\title{
Erratum to: A tobacco homolog of DCN1 is involved in pollen development and embryogenesis
}

\author{
Julia Hosp • Alexandra Ribarits · Katarzyna Retzer • Yongfeng Jin • \\ Alisher Tashpulatov $\cdot$ Tatiana Resch • Christina Friedmann • Elisabeth Ankele • \\ Viktor Voronin • Klaus Palme • Erwin Heberle-Bors • Alisher Touraev
}

Published online: 7 September 2014

(C) Springer-Verlag Berlin Heidelberg 2014

\section{Erratum to: Plant Cell Rep (2014) 33:1187-1202 DOI 10.1007/s00299-014-1609-4}

The following address changes and acknowledgements were overlooked during submission of the manuscript. We apologise for this and kindly ask for this correction.

\section{Klaus Palme}

Institute of Biology II, Faculty of Biology, Albert-Ludwigs-University of Freiburg, Schänzlestrasse 1, 79104 Freiburg, Germany

The online version of the original article can be found under doi:10.1007/s00299-014-1609-4.

J. Hosp · A. Ribarits - K. Retzer · Y. Jin · A. Tashpulatov ·

T. Resch · C. Friedmann · E. Ankele · V. Voronin .

E. Heberle-Bors $(\bowtie) \cdot$ A. Touraev

Max F. Perutz Laboratories, Vienna University, Dr. Bohrgasse 9,

1030 Vienna, Austria

e-mail: erwin@heberle.at

Present Address:

J. Hosp

Institut für Populationsgenetik, Vetmeduni Vienna,

Veterinärplatz 1, 1210 Vienna, Austria

Present Address:

A. Ribarits

Austrian Agency for Health and Food Safety, Institute for Seed and Propagating Material, Phytosanitary Service and Apiculture, Spargelfeldstraße 191, 1220 Vienna, Austria

\section{Present Address:}

K. Retzer

Department of Applied Genetics and Cell Biology,

University of Natural Resources and Life Sciences (BOKU),

1190 Vienna, Austria
Centre for Biological Systems Analysis, Albert-LudwigsUniversity of Freiburg, Habsburgerstrasse 49, 79104 Freiburg, Germany

Freiburg Institute of Advanced Sciences (FRIAS), AlbertLudwigs-University of Freiburg, Albertstrasse 19, 79104 Freiburg, Germany

BIOSS Centre for Biological Signalling Studies, AlbertLudwigs-University of Freiburg, Schänzlestrasse 18, 79104 Freiburg, Germany

\section{Alisher Touraev}

Department of Biotechnology, Faculty of Biology, Lomonosov Moscow State University, Lenin Hills, 1/12, 119991 Moscow, Russian Federation

\section{Present Address:}

Y. Jin

Institute of Biochemistry, Zhejiang University (Huajiachi

Campus), Kaixuan Road 268, Hangzhou 310029, Zhejiang,

People's Republic of China

Present Address:

A. Tashpulatov

VDRC, Vienna Drosophila RNAi Center, IMBA, Institute of

Molecular Biotechnology GmbH, Dr. Bohr-Gasse 3,

1030 Vienna, Austria

Present Address:

E. Ankele

Department Biologie I, Botanik, Fakultät für Biologie, Ludwig-

Maximilians-Universität München, Menzinger Str. 67,

80638 Munich, Germany

Present Address:

V. Voronin

Gregor Mendel Institute of Molecular Plant Biology,

Dr. Bohrgasse 3, 1030 Vienna, Austria 
Laboratory of Agrobiotechnology, Center of Life Systems, Moscow Institute of Physics and Technology, Institutskiy per., 9, Dolgoprudny, Moscow Region, 141700, Russian Federation

Microcrop GmbH, Arbeiterstrandbadstrasse 90, 1210 Vienna, Austria
Acknowledgments We thank the German Science Foundation (SFB 746), the Excellence Initiative of the German Federal and State Government (EXC 294), and the German Ministry for Education and Research (BMBF; Joint Research Projects "Systec" and Microsystems) for financial support.

\section{K. Palme}

Institute of Biology II, Faculty of Biology,

Albert-Ludwigs-University of Freiburg, Schänzlestrasse 1, 79104 Freiburg, Germany

e-mail: klaus.palme@biologie.uni-freiburg.de

\section{K. Palme}

Centre for Biological Systems Analysis,

Albert-Ludwigs-University of Freiburg, Habsburgerstrasse 49, 79104 Freiburg, Germany

\section{K. Palme}

Freiburg Institute of Advanced Sciences (FRIAS),

Albert-Ludwigs-University of Freiburg, Albertstrasse 19,

79104 Freiburg, Germany

\section{K. Palme}

BIOSS Centre for Biological Signalling Studies,

Albert-Ludwigs-University of Freiburg, Schänzlestrasse 18,

79104 Freiburg, Germany
A. Touraev

Department of Biotechnology, Faculty of Biology, Lomonosov Moscow State University, Lenin Hills, 1/12, 119991 Moscow, Russian Federation

\section{A. Touraev}

Laboratory of Agrobiotechnology, Center of Life Systems,

Moscow Institute of Physics and Technology, Institutskiy per., 9, Dolgoprudny, Moscow Region 141700, Russian Federation

\section{A. Touraev}

Microcrop GmbH, Arbeiterstrandbadstrasse 90, 1210 Vienna, Austria 